\title{
Community-Based Assessment of Knowledge, Attitudes, and Practices Towards COVID-19: an Epidemiological Survey in Kurdistan Region, Iraq
}

\author{
Zhino Raouf Ali \\ Health Development and Training Center \\ Directorate of Health- Slemani Administration \\ Ministry of Health \\ Sulaimani, Iraq \\ zhino.hdtc@gmail.com \\ Aram Salih Mohammed Amin Kamali \\ Planning Dept. \\ Directorate of Health- Garmian Administration \\ Ministry of Health \\ Kalar, Sulaimani, Iraq \\ aram.sharwani@gmail.com \\ Azad Karim Ahmed \\ Cardiac Specialty Hospital \\ Directorate of Health- Slemani Administration \\ Ministry of Health \\ Sulaimani, Iraq \\ azadsg4@gmail.com \\ Chiman Jamal ahmed \\ Thalassemia and congenital blood disorders center \\ Directorate of Health- Slemani Administration \\ Ministry of Health \\ Sulaimani, Iraq \\ Cheeman.jamal@gmail.com
}

\author{
Bayan Omar Sharif \\ Health Development and Training Center \\ Directorate of Health- Slemani Administration \\ Ministry of Health \\ Sulaimani, Iraq \\ Omerbayan82@gmail.com \\ Varin Tofiq Abbas \\ Infection Control Dept. \\ Directorate of Health- Slemani Administration \\ Ministry of Health \\ Sulaimani, Iraq \\ varin7815@yahoo.com \\ Shaho Osman Mahmood \\ Surgical Teaching Hospital \\ Directorate of Health- Slemani Administration \\ Ministry of Health \\ Sulaimani, Iraq \\ Shahohusman@yahoo.co.uk
Sarwat Ahmed Abdulqader
Dialysis Center
Directorate of Health- Slemani Administration
Ministry of Health
Sulaimani, Iraq \\ sarwat.abdulqader@gmail.com
}

\section{Article Info \\ Volume 5 - Issue 2 -December 2020 \\ DOI: \\ 10.24017/science.2020.2.1}

\section{Article history:}

Received: 30 August 2020

Accepted: 29 September 2020

\begin{abstract}
Covid-19 considered as a pandemic disease and spread very rapidly, the people are needed to respond to this danger situation without becoming complacent because still there is no vaccine for it. The sample of this cross - sectional descriptive study was consisted of 1250 Kurdish male and female, their ages ranged between 14- 76 years. The main objective of the study is conducted to assess the people's knowledge, attitude, and practice (KAP study) toward COVID19. The data were collected during the period of April 4 up to
\end{abstract}




\section{Keywords: \\ COVID-19, Pandemic, Knowledge, Attitude, Practices, Kurdish, Kurdistan region}

June 2020. A self- constructed questionnaire was used for data collection and it was designed according guidelines, literatures, and articles concerning COVID-19; which consisted of 40 variables. The researchers made google form for the questionnaire and distributed by local residents of Kurdistan region. Relying on the authors' networks, and sent by (Facebook, Instagram, WhatsApp, and Telegram). Therefore, the data analyzed through the using of the (SPSS, version 23). A p value of $\leq 0.05$ was considered statistically significant. The findings of the current study concluded that the main way for obtaining knowledge of the participants was social media and it was much more than ministry of health, WHO and CDC together. Also, the study indicated that the age group of 21-30 years old has a high score of knowledge and attitude while the age group of 31-40 years old has a high score of practical issues regarding COVID-19. As well as, the female employer by Bachelor degree with barely sufficient financial status in urban area had a high score of KAP concerning COVID-19, lastly the present study observed a strong positive correlation between KAP toward coronavirus among the participants. The study recommended to ministry of health and health sectors to present much more scientific healthy issues to improve the knowledge, attitude, and practice of the Kurdish citizens concerning coronavirus because the health care workers are much more dependable scientifically than social media toward COVID-19 to prevent rapid transmission and control this serious situation.

Copyright (C) 2020 Kurdistan Journal of Applied Research. All rights reserved.

\section{INTRODUCTION}

An emerging respiratory disease; Coronavirus disease is abbreviated as COVID-19 or coronavirus disease 2019 that is caused by a novel coronavirus and was first discovered in December 2019 in Wuhan, China. High body temperature, myalgia, and dry cough are the main clinical symptoms of this highly infectious disease [1]. This disease has spread very rapidly, and by mid-February, 2020, the virus had reached nearly 30 countries altogether. In response to this serious condition, Coronaviruses can cause multiple system infections in various animals and mainly respiratory tract infections in humans, such as severe acute respiratory syndrome (SARS) and Middle East respiratory syndrome (MERS) [2, 3]. In 2003 when the SARS emerged it was detected that any newly emerging zoonotic coronaviruses has the probable to transmit from person to person, especially in healthcare settings, and lead to severe human sicknesses [4]. One of the first oldest and most effective tools for controlling communicable and epidemic disease outbreaks is quarantine [5]; It was implemented positively as an effective measure during the epidemic disease of SARS in 2003 [6]. Quarantine is an important element of pandemic disease ideas. It means the restriction of movement of persons who are supposed to have been exposed to an infectious disease but they are still healthy and were not infected or because they are still in the incubation period [7]. Designing a social distance or self-isolation is very useful to reduce communications and interactions between people in a wider community, so this serious diseases transmitted by respiratory droplets and the individuals may be infected but have no signs and symptoms or are not diagnosed yet. So it is required from the people to immediately keep away from people and implement social distancing because it is one of the factors that lead to reduce transmission of the disease [8]. Keeping away of infected people with infectious diseases from non-infected people is called Separation. Most governments were in the practice of evacuating 
of their citizens, and these people will be under supervision for fourteen days quarantine up on arrival in their home country to decrease viral shedding in the first few days of illness to prevent transmission of this serious disease [9]. Entering and Coping with Isolation is an important of the fundamental response to the coronavirus pandemic is self-isolation through humans with signs and symptoms or who have been in close contact with contaminated persons. While massive wide variety of studies offer clear proof of terrible effects of psychological problems concerning self-isolation but it can lead to compromise and manage the spread of infectious diseases [10]. Coronavirus is that serious disease that could constrain a significant number of individuals to remaining at household and plunking down more noteworthy than peoples ordinarily do. Normally exercise has the two focal points for both brain and body; likewise can give daily life pastimes and is an approach to keep on being in contact with loved ones and bringing down the opportunity of gloom. Smoking is another demonstration that builds the opportunity the transmission of coronavirus from hand to mouth or coming about, because of sullied cigarettes when contact with lips. For example, water pipes frequently contain the sharing of mouth segments and hoses, which quickly transmitted the coronavirus among resident and social settings. Iraq has affirmed instances of COVID-19 inside its outskirts. Iraq detailed its first case in late February and mid-March; wellbeing specialists had restricted significant open occasions, suspended schools, and shut shopping centers and assembling places [3]. At the beginning time of the coronavirus disease the Iraqi Ministry of Health had confirmed more than 150 cases of COVID-19 in Iraq; more than 10 of them were fatalities; and less than 50 patients who have recovered from the corona virus; because of this risky situation both of government of Iraq and the Kurdistan Regional Government have started strict movement restrictions to try to further stem any transmission of the virus. Kurdistan region has been hit by the COVID-19 epidemic. Some unprecedented measures have been adopted to control the COVID-19 transmission in Kurdistan with other cities of Iraq, including the suspension of public transportation, the closing of public spaces, close management of communities, and isolation and care for infected people and suspected cases [11]. According to ministry of health in Kurdistan region about 12450 cases have registered since first cases on February, 2020 till now and more than 490 cases have died [12]. following the guidelines and steps for controlling coronavirus is very important, so lack of knowledge, attitudes, and practices (KAP) lead to increase the rate of fear among population and it influenced on their health, while the researchers decided to do this study therefore the aim of this study was assessing of people's knowledge, attitude and practices toward COVID19.

\section{Study design and setting}

\section{METHODS AND MATERIALS}

A quantitative approach conducted using descriptive cross - sectional study in Kurdistan Regional Government (KRG) - Iraq, among Kurdish residency in four governorates (Erbil, Slemani, Duhok and Halabja) toward COVID-19 from April 4 to August 20.

\section{Sample of the study}

Simple random sampling technique was applied to collect data from 1250 people. The respondents were sent via social media bilingual (English-Kurdish) questionnaires. Primary questionnaire was developed after comprehensive literature review and articles later translated to Kurdish language by a native speaker. A final version was agreed on, which was validated in a pilot that tested the clarity and relevance of the statements by ten experts.

\section{Variables}

The dependent variables were knowledge, attitudes, and practices toward COVID-19 outbreaks. Independent variables included age, gender, residential area, educational level, occupation, and monthly income.

\section{Data sources/ measurement}

In addition to the demographic data, eleven questions were asked to assess knowledge; twelve questions explored attitudes and ten questions focused on practices towards COVID-19 outbreaks. The questionnaire was consisted of short answer of choosing the best answer and 
multiple choice formats. The first hundred forms were completed and resent back for the pilot study was conducted with Cronbach alpha $(\mathrm{r}=0.72)$.

\section{Data collection and Analysis}

According to Kurdistan - Ministry of Health, and in order to prevent person-to-person contact, bilingual online questionnaire developed and emailed to participants through Google forms. Corresponding researcher was on-call during the data collection for answering any probable question. This Web-based format was selected for lower infection risk.

For data analysis, the mean, standard deviation, and interquartile range were calculated. Kolmogorov-Smirnov test and Q-Q plot used for checking normal distribution of data with an estimated 95\% confidence interval. A t-test, Mann-Whiney test, and ANOVA and KruskalWallis test used to compare between the subgroups based on test for the normal distribution of data. The questionnaire was labeled and coded by the researchers; data was reviewed for accuracy and completeness and analyzed using SPSS 23 (IBM Corp. Released 2013. Also using chi-square test, for each domain scores of the study participants were categorized into two categories: good and poor.

\section{RESULTS}

Out of 1250 participants the minimum age was 14 years, while the maximum age was 76 years old with mean age (32.88 (9.973). Table (1) Showed that the age of the participants were between ( $\leq 20-\geq 41)$ years, (54.6\%) of them were female, nearly half of them were graduated from university which were the highest level (46.2\%) and lowest level of their education was graduated from primary school (2.6\%), (67.2\%) of them were from urban area, only (5.1\%) of them were from rural area, and highest rate of their occupation was governmental employ (56.3\%). (65.8\%) of their economic status were barely sufficient and (83.8\%) of them were not smoke any types of smoking such as cigarette and Hookah.

Table 1: Socio- demographic Descriptive

\begin{tabular}{|c|c|c|c|}
\hline Variables & Categories & $\mathbf{F}$ & $(\%)$ \\
\hline \multirow[t]{4}{*}{ Age (Years) } & $\leq 20$ & 126 & 10.1 \\
\hline & $21-30$ & 436 & 34.9 \\
\hline & $31-40$ & 432 & 34.6 \\
\hline & $\geq 41$ & 256 & 20.5 \\
\hline \multirow[t]{2}{*}{ Gender } & Male & 567 & 45.4 \\
\hline & Female & 683 & 54.6 \\
\hline \multirow[t]{3}{*}{ Residential Area } & Urban & 840 & 67.2 \\
\hline & Sub- urban & 346 & 27.7 \\
\hline & Rural & 64 & 5.1 \\
\hline \multirow[t]{6}{*}{ Educational Level } & Primary School & 32 & 2.6 \\
\hline & Secondary School & 145 & 11.6 \\
\hline & Diploma & 305 & 24.4 \\
\hline & Bachelor & 578 & 46.2 \\
\hline & Master & 140 & 11.2 \\
\hline & $\mathrm{PhD}$ & 50 & 4.0 \\
\hline \multirow[t]{6}{*}{ Occupation } & Student & 243 & 19.4 \\
\hline & Governmental Employ & 704 & 56.3 \\
\hline & Self-employ & 139 & 11.1 \\
\hline & Housewife & 53 & 4.2 \\
\hline & Jobless & 102 & 8.2 \\
\hline & Retired & 9 & .7 \\
\hline \multirow[t]{3}{*}{ Monthly Income } & Sufficient & 361 & 28.9 \\
\hline & Barely sufficient & 822 & 65.8 \\
\hline & Insufficient & 67 & 5.4 \\
\hline \multirow[t]{4}{*}{ Which one of these do you smoke? } & Cigarette & 136 & 10.9 \\
\hline & Hookah & 45 & 3.6 \\
\hline & Both of them & 22 & 1.8 \\
\hline & None of them & 1047 & 83.8 \\
\hline
\end{tabular}


Table 2: Sources of knowledge regarding COVID-19

\begin{tabular}{|c|c|c|c|}
\hline Variable & Categories & $\mathbf{F}$ & $\%$ \\
\hline \multirow{10}{*}{$\begin{array}{l}\text { Which sources do you mostly } \\
\text { use for obtaining dependable } \\
\text { information } \\
\text { Coronavirus? }\end{array}$} & Social media & 456 & 36.48 \\
\hline & TV & 175 & 14 \\
\hline & TV / Social media & 124 & 9.9 \\
\hline & TV / Radio \& Social media & 30 & 2.4 \\
\hline & Official websites of WHO & 118 & 9.4 \\
\hline & Ministry of health & 105 & 8.4 \\
\hline & Ministry of health / WHO & 175 & 14 \\
\hline & Official websites of (WHO \& CDC) & 37 & 3 \\
\hline & College of nursing and medicine & 46 & 3.68 \\
\hline & Ministry of health / WHO \& CDC & 28 & 2.24 \\
\hline
\end{tabular}

Table (2) demonstrates that the highest source of participant's knowledge was obtained through social media (36.48\%) followed by the lowest source of participant's knowledge, which they obtained through (Ministry of health / WHO and CDC) together.

Table 3: Knowledge of participants regarding COVID-19

\begin{tabular}{|c|c|c|c|c|}
\hline No. & Items & Response & $\mathbf{F}$ & $\%$ \\
\hline \multirow[t]{3}{*}{ K1 } & \multirow{3}{*}{$\begin{array}{c}\text { If you experienced the symptoms } \\
\text { of COVID-19, what would you } \\
\text { do? }\end{array}$} & Call health care services "122" & 964 & 77.1 \\
\hline & & $\begin{array}{l}\text { Stay at home and avoid contact with } \\
\text { others }\end{array}$ & 261 & 20.9 \\
\hline & & I do not take it too seriously & 25 & 2.0 \\
\hline \multirow[t]{3}{*}{ K2 } & \multirow{3}{*}{$\begin{array}{l}\text { If someone contacted with infected } \\
\text { person with the COVID-19; what } \\
\text { should be do immediately? }\end{array}$} & Immediately isolated in the quarantine & 1192 & 95.4 \\
\hline & & Use medication & 32 & 2.6 \\
\hline & & Does not take care to prevent fear & 26 & 2.1 \\
\hline \multirow[t]{3}{*}{ K3 } & \multirow{3}{*}{$\begin{array}{l}\text { Are cigarette and hookah (Shisha) } \\
\text { smokers more at risk of getting } \\
\text { infected by Coronavirus infection? }\end{array}$} & Yes & 1027 & 82.2 \\
\hline & & I don't know & 171 & 13.7 \\
\hline & & No & 52 & 4.2 \\
\hline \multirow[t]{3}{*}{ K4 } & \multirow{3}{*}{$\begin{array}{l}\text { Is Coronavirus transmitted from } \\
\text { materials that are imported from } \\
\text { other countries? }\end{array}$} & Yes & 634 & 50.7 \\
\hline & & No & 355 & 28.4 \\
\hline & & I don't know & 261 & 20.9 \\
\hline \multirow[t]{3}{*}{ K5 } & \multirow{3}{*}{$\begin{array}{l}\text { Are there enough instructions for } \\
\text { people recovering from Corona } \\
\text { virus after being discharged from } \\
\text { the hospital? }\end{array}$} & Yes & 829 & 66.3 \\
\hline & & I don't know & 332 & 26.6 \\
\hline & & No & 89 & 7.1 \\
\hline \multirow[t]{3}{*}{ K6 } & \multirow{3}{*}{$\begin{array}{c}\text { Are the TV channels continuously } \\
\text { spreading bad news about } \\
\text { increasing numbers }\end{array}$} & Yes & 1078 & 86.2 \\
\hline & & No & 144 & 11.5 \\
\hline & & I don't know & 28 & 2.2 \\
\hline \multirow[t]{3}{*}{ K7 } & \multirow{3}{*}{$\begin{array}{l}\text { Does drinking alcohol protect you } \\
\text { from Corona virus? }\end{array}$} & No & 988 & 79.0 \\
\hline & & I don't know & 208 & 16.6 \\
\hline & & Yes & 54 & 4.3 \\
\hline \multirow[t]{3}{*}{ K8 } & \multirow{3}{*}{$\begin{array}{l}\text { Is Corona virus transmitted from } \\
\text { the mosquito? }\end{array}$} & I don't know & 585 & 46.8 \\
\hline & & No & 522 & 41.8 \\
\hline & & Yes & 143 & 11.4 \\
\hline \multirow[t]{3}{*}{ K9 } & \multirow{3}{*}{$\begin{array}{l}\text { Does self-exposure to sunlight or } \\
\text { any temperature higher than } 25^{\circ} \mathrm{c} \\
\text { protect you from Coronavirus? }\end{array}$} & Yes & 595 & 47.6 \\
\hline & & No & 381 & 30.5 \\
\hline & & I don't know & 274 & 21.9 \\
\hline \multirow[t]{4}{*}{ K10 } & \multirow{4}{*}{$\begin{array}{l}\text { Which time you should wash your } \\
\text { hands? }\end{array}$} & Before and after meal & 17 & 1.4 \\
\hline & & After using WC & 28 & 2.2 \\
\hline & & After touching money or animals & 11 & .9 \\
\hline & & All of them & 1194 & 95.5 \\
\hline \multirow[t]{3}{*}{ K11 } & \multirow{3}{*}{$\begin{array}{l}\text { Is daily Physical activity important } \\
\text { according to ages and abilities } \\
\text { during the outbreak of } \\
\text { Coronavirus? }\end{array}$} & Yes & 876 & 70.1 \\
\hline & & To an extent & 294 & 23.5 \\
\hline & & No & 80 & 6.4 \\
\hline
\end{tabular}


Table (3) showed that the participants have recorded good knowledge regarding COVID-19 and they were responded the questions correctly in (K1, K2, K3, K5, K6, K7, K10, K11).

Table 4: Participant's Attitude regarding COVID-19

\begin{tabular}{|c|c|c|c|c|}
\hline No & Items & $\begin{array}{l}\text { Yes } \\
\text { F (\%) }\end{array}$ & $\begin{array}{l}\text { To an extent } \\
\text { F (\%) }\end{array}$ & $\begin{array}{c}\text { No } \\
\text { F (\%) }\end{array}$ \\
\hline A1 & $\begin{array}{l}\text { In your belief, cleaning your hands and } \\
\text { preventing them from touching your face is } \\
\text { the best way to reduce the risk of spreading } \\
\text { Coronavirus. }\end{array}$ & 1017 (81.4) & $197(15.8)$ & $36(2.9)$ \\
\hline A2 & $\begin{array}{l}\text { According to your point of view; does not } \\
\text { exist or it doesn't has no a scientific base it } \\
\text { mean it is a biological war? }\end{array}$ & $200(16.0)$ & $400(32.0)$ & $650(52.0)$ \\
\hline A3 & $\begin{array}{l}\text { Do you think keeping social distance in the } \\
\text { community is important to the patient even } \\
\text { after recovering from coronavirus and leaving } \\
\text { the hospital? }\end{array}$ & $812(65.0)$ & 309 (24.7) & $129(10.3)$ \\
\hline A4 & $\begin{array}{l}\text { Do you believe that using masks only; is } \\
\text { enough to keep you safe from coronavirus? }\end{array}$ & $54(4.3)$ & 287 (23.0) & 909 (72.7) \\
\hline A5 & $\begin{array}{l}\text { Do you believe that using gloves only is } \\
\text { enough to keep you safe (away) from } \\
\text { coronavirus? }\end{array}$ & $45(3.6)$ & 272 (21.8) & $933(74.6)$ \\
\hline A6 & $\begin{array}{l}\text { Do you think that it is a good idea to prevent } \\
\text { TV channels and social media from publishing } \\
\text { unpleasant and frightening news about the } \\
\text { number of people affected and died by } \\
\text { coronavirus? }\end{array}$ & $650(52.0)$ & 354 (28.3) & 246 (19.7) \\
\hline A7 & $\begin{array}{l}\text { Do you believe those who took vaccines in } \\
\text { their childhood against Tuberculosis (TB) will } \\
\text { not be affected by coronavirus or will be } \\
\text { easily recovered from it? }\end{array}$ & 359 (28.7) & 647 (51.8) & 244 (19.5) \\
\hline A8 & $\begin{array}{l}\text { Do you believe in the advice about curfew and } \\
\text { staying at home? }\end{array}$ & 872 (69.8) & 264(21.1) & $114(9.1)$ \\
\hline A9 & $\begin{array}{l}\text { In your opinion, will keeping people indoors } \\
\text { by force harm people in terms of their } \\
\text { finances? }\end{array}$ & 898 (71.8) & $313(25.0)$ & 39 (3.1) \\
\hline A10 & $\begin{array}{l}\text { Do you think that you have to wash your } \\
\text { hands in a healthy way that is not less than } \\
\text { twenty (20) seconds? }\end{array}$ & 1047 (83.8) & 143 (11.4) & $60(4.8)$ \\
\hline A11 & $\begin{array}{l}\text { Do you think that the health guidelines have } \\
\text { been useful for protecting you from } \\
\text { coronavirus? }\end{array}$ & $1025(82.0)$ & 193 (15.4) & $32(2.6)$ \\
\hline A12 & $\begin{array}{l}\text { Do you think that social media has caused you } \\
\text { any confusion regarding Coronavirus? }\end{array}$ & 437 (35.0) & 494 (39.5) & 319 (25.5) \\
\hline
\end{tabular}

Table (4) demonstrated that the most participants were believed and answered most of the questions correctly especially in (A1, A3, A4, A5, A6, A8, A9, A10, and A11).

Table 5: Practice of participants regarding COVID-19

\begin{tabular}{llccc}
\hline No & \multicolumn{1}{c}{ Items } & $\begin{array}{c}\text { Always } \\
\mathbf{F ~ ( \% )}\end{array}$ & $\begin{array}{c}\text { Sometimes } \\
\mathbf{F ~ ( \% )}\end{array}$ & $\begin{array}{c}\text { Never } \\
\mathbf{F ~ ( \% )}\end{array}$ \\
\hline P1 & $\begin{array}{l}\text { Did you try to stay at home and prevent } \\
\text { visiting your relatives and friends to protect } \\
\text { yourself and others from Coronavirus? }\end{array}$ & $884(70.7$ & $346(27.7)$ & $20(1.6)$ \\
\hline P2 & Do you use mask and gloves when you go out? & $521(41.7)$ & $578(46.2)$ & $151(12.1)$ \\
\hline P3 & $\begin{array}{l}\text { Do you wash every part of your hands such as } \\
\text { palm, thumb, wrist, under nails and fingers } \\
\text { very well by soap and water when you return }\end{array}$ & $923(73.8)$ & $296(23.7)$ & $31(2.5)$ \\
\hline
\end{tabular}




\begin{tabular}{|c|c|c|c|c|}
\hline & home? & & & \\
\hline P4 & $\begin{array}{l}\text { Do you do physical activity according your age } \\
\text { and ability when you at home because of } \\
\text { Coronavirus? }\end{array}$ & $220(17.6)$ & 765 (61.2) & $265(21.2)$ \\
\hline P5 & $\begin{array}{l}\text { Do you destroy those masks and gloves after } \\
\text { using from a suitable place? }\end{array}$ & 987 (79.0) & 198 (15.8) & $65(5.2)$ \\
\hline P6 & $\begin{array}{l}\text { Did you design a healthy suitable schedule for } \\
\text { your daily life until you will spend your time } \\
\text { far away from stress and fear? }\end{array}$ & $490(39.2)$ & 579 (46.3) & $181(14.5)$ \\
\hline P7 & $\begin{array}{l}\text { Do you follow that bad and sadness news } \\
\text { which presented from TV and Internet } \\
\text { regarding increasing number of death and new } \\
\text { case of Corona virus? }\end{array}$ & 244 (19.5) & $682(54.6)$ & 324 (25.9) \\
\hline P8 & $\begin{array}{l}\text { Did you try to prevent hand shaking with } \\
\text { everybody to protect yourself and others from } \\
\text { Corona virus? }\end{array}$ & $775(62.0)$ & 411 (32.9) & $64(5.1)$ \\
\hline P9 & $\begin{array}{l}\text { Do you wash your hand by soap and water or } \\
\text { alcohol immediately when you cough or } \\
\text { sneeze on your hand? }\end{array}$ & 779 (62.3) & 394 (31.5) & $77(6.2)$ \\
\hline P10 & $\begin{array}{l}\text { Did you annually inject the flu vaccination } \\
\text { against seasonal flu? }\end{array}$ & 165 (13.2) & 356 (28.5) & 729 (58.3) \\
\hline
\end{tabular}

Table (5) showed that the participants were always practiced in a healthy way to protect their health toward coronavirus especially in (P1, P3, P5, P8, and P9) except (P2, P4, P6, P7 and P10).

Table 6: Correlation Between Knowledge, Attitude and practice

\begin{tabular}{lcc}
\hline Variables & Rho & P-value \\
\hline Knowledge, Attitude & .296 & $0.000^{*}$ \\
\hline Knowledge, Practice & .254 & $0.000^{*}$ \\
\hline Attitude, Practice & .287 & $0.000^{*}$ \\
\hline${ }^{*}$ Statistically significant at $\mathbf{p}<\mathbf{0 . 0 5}$ & & \\
\hline
\end{tabular}

A correlation between different domains of questionnaire was also assessed. A strong positive correlation was observed between knowledge, attitude, and practice Table (6).

Table 7: Mean score with respect to demographics

\begin{tabular}{|c|c|c|c|c|c|c|c|}
\hline Variable & & $\begin{array}{l}\text { K score } \\
1.54 \\
(0.22) \\
\text { Mean } \\
\text { Rank }\end{array}$ & סِ & $\begin{array}{l}\text { A score } \\
1.53 \\
(0.21) \\
\text { Mean } \\
\text { Rank }\end{array}$ & 苞 & $\begin{array}{l}\text { P score } \\
1.33 \\
(0.31) \\
\text { Mean } \\
\text { Rank }\end{array}$ & ه্ \\
\hline \multirow[t]{4}{*}{ Age group } & $\leq 20$ & 570.57 & \multirow[t]{4}{*}{.149} & 623.01 & \multirow[t]{4}{*}{.070} & 479.12 & \multirow[t]{4}{*}{.000} \\
\hline & $21-30$ & 613.21 & & 590.26 & & 605.12 & \\
\hline & $31-40$ & 639.38 & & 645.94 & & 613.25 & \\
\hline & $\geq 41$ & 650.04 & & 652.26 & & 752.91 & \\
\hline \multirow[t]{2}{*}{ Gender } & Male & 607.14 & \multirow[t]{2}{*}{.099} & 593.58 & \multirow[t]{2}{*}{.004} & 569.31 & \multirow[t]{2}{*}{.000} \\
\hline & Female & 640.74 & & 652.00 & & 672.15 & \\
\hline \multirow{3}{*}{$\begin{array}{l}\text { Residential } \\
\text { Area }\end{array}$} & Urban & 637.03 & \multirow[t]{3}{*}{.057} & 643.56 & \multirow[t]{3}{*}{.004} & 687.26 & \multirow[t]{3}{*}{.000} \\
\hline & Sub- urban & 615.26 & & 605.17 & & 509.76 & \\
\hline & Rural & 529.58 & & 498.39 & & 440.66 & \\
\hline \multirow{6}{*}{$\begin{array}{l}\text { Educational } \\
\text { Level }\end{array}$} & Primary School & 449.05 & \multirow[t]{6}{*}{.000} & 640.50 & \multirow[t]{6}{*}{.339} & 602.28 & \multirow[t]{6}{*}{.000} \\
\hline & $\begin{array}{l}\text { Secondary } \\
\text { School }\end{array}$ & 494.55 & & 573.46 & & 617.03 & \\
\hline & Diploma & 630.45 & & 652.95 & & 606.74 & \\
\hline & Bachelor & 645.75 & & 629.68 & & 602.60 & \\
\hline & Master & 692.13 & & 600.34 & & 703.86 & \\
\hline & $\mathrm{PhD}$ & 667.27 & & 621.54 & & 824.64 & \\
\hline
\end{tabular}




\begin{tabular}{|c|c|c|c|c|c|c|c|}
\hline \multirow[t]{6}{*}{ Occupation } & Student & 617.58 & \multirow[t]{6}{*}{.000} & 589.44 & \multirow[t]{6}{*}{.020} & 558.77 & \multirow[t]{6}{*}{.000} \\
\hline & $\begin{array}{l}\text { Governmental } \\
\text { Employ }\end{array}$ & 665.04 & & 646.61 & & 672.02 & \\
\hline & Self-employ & 573.23 & & 561.63 & & 590.04 & \\
\hline & Housewife & 430.17 & & 625.08 & & 517.58 & \\
\hline & Jobless & 534.40 & & 631.75 & & 552.28 & \\
\hline & Retired & 736.94 & & 866.11 & & 800.94 & \\
\hline \multirow{3}{*}{$\begin{array}{l}\text { Monthly } \\
\text { Income }\end{array}$} & Sufficient & 658.31 & \multirow[t]{3}{*}{.000} & 654.54 & \multirow[t]{3}{*}{.000} & 699.05 & \multirow[t]{3}{*}{.000} \\
\hline & Barely sufficient & 624.06 & & 625.98 & & 601.64 & \\
\hline & Insufficient & 466.43 & & 463.07 & & 521.89 & \\
\hline
\end{tabular}

Table (7) indicated that highest mean score of (KAP) presented in female, retired, urban area in sufficient income in age group of $(\geq 41)$ years, but master degree for knowledge, Diploma for attitude and PhD degree for practices have a high mean of score.

Table 8: Categorization of study participants score on KAP domains

\begin{tabular}{|c|c|c|c|c|c|c|c|c|c|}
\hline \multirow{2}{*}{ Variable } & \multicolumn{2}{|c|}{ Knowledge } & \multirow{2}{*}{$\frac{\tilde{\sigma}}{\tilde{\Sigma}}$} & \multicolumn{2}{|c|}{ Attitude } & \multirow{2}{*}{$\frac{\sigma}{\tilde{\delta}}$} & \multicolumn{2}{|c|}{ Practice } & \multirow{2}{*}{$\underset{\delta}{\tilde{\sigma}}$} \\
\hline & Poor & Good & & Poor & Good & & Poor & Good & \\
\hline \multicolumn{10}{|c|}{ Age group } \\
\hline$\leq \mathbf{2 0}$ & 3 & 117 & \multirow[t]{4}{*}{0.059} & 3 & 122 & \multirow[t]{4}{*}{0.353} & 44 & 82 & \multirow{4}{*}{$\begin{array}{r}0.00 \\
0\end{array}$} \\
\hline $21-30$ & 5 & 426 & & 10 & 420 & & 80 & 356 & \\
\hline $31-40$ & 3 & 424 & & 16 & 409 & & 74 & 358 & \\
\hline$\geq 41$ & 1 & 253 & & 1 & 252 & & 24 & 232 & \\
\hline \multicolumn{10}{|c|}{ Gender } \\
\hline Male & 9 & 550 & \multirow[t]{2}{*}{0.038} & 18 & 536 & \multirow[t]{2}{*}{0.093} & 135 & 432 & \multirow{2}{*}{$\begin{array}{r}0.00 \\
0 \\
\end{array}$} \\
\hline Female & 3 & 670 & & 12 & 667 & & 87 & 596 & \\
\hline \multicolumn{10}{|c|}{ Residential Area } \\
\hline Urban & 7 & 821 & \multirow[t]{3}{*}{0.461} & 14 & 818 & \multirow[t]{3}{*}{0.000} & 100 & 740 & \multirow{3}{*}{$\begin{array}{r}0.00 \\
0\end{array}$} \\
\hline Sub- urban & 4 & 339 & & 9 & 330 & & 96 & 250 & \\
\hline Rural & 1 & 60 & & 7 & 55 & & 26 & 38 & \\
\hline \multicolumn{10}{|c|}{ Educational Level } \\
\hline P. School & 1 & 30 & \multirow[t]{6}{*}{0.292} & 2 & 30 & \multirow[t]{6}{*}{0.972} & 6 & 26 & \multirow{6}{*}{$\begin{array}{r}0.00 \\
1\end{array}$} \\
\hline S. School & 2 & 138 & & 2 & 139 & & 32 & 113 & \\
\hline Diploma & 3 & 297 & & 8 & 296 & & 59 & 246 & \\
\hline Bachelor & 5 & 567 & & 12 & 556 & & 112 & 466 & \\
\hline Master & 0 & 140 & & 5 & 133 & & 10 & 130 & \\
\hline PhD & 1 & 48 & & 1 & 49 & & 3 & 47 & \\
\hline \multicolumn{10}{|c|}{ Occupation } \\
\hline Student & 9 & 225 & \multirow[t]{6}{*}{0.015} & 7 & 231 & \multirow[t]{6}{*}{0.955} & 60 & 183 & \multirow{6}{*}{$\begin{array}{r}0.13 \\
0\end{array}$} \\
\hline $\begin{array}{l}\text { Employment } \\
\mathrm{s}\end{array}$ & 1 & 696 & & 16 & 682 & & 86 & 618 & \\
\hline Self-employ & 1 & 138 & & 2 & 131 & & 36 & 103 & \\
\hline Housewife & 1 & 52 & & 2 & 51 & & 12 & 41 & \\
\hline Jobless & 0 & 100 & & 3 & 99 & & 28 & 74 & \\
\hline Retired & 0 & 9 & & 0 & 9 & & 0 & 9 & \\
\hline \multicolumn{10}{|c|}{ Monthly Income } \\
\hline Sufficient & 2 & 355 & 0.009 & 8 & 352 & 0.447 & 39 & 322 & 0.00 \\
\hline $\begin{array}{l}\text { Barely } \\
\text { sufficient }\end{array}$ & 6 & 802 & & 19 & 790 & & 154 & 668 & 0 \\
\hline Insufficient & 4 & 63 & & 3 & 61 & & 29 & 38 & \\
\hline Total & 12 & 1220 & & 30 & 1203 & & 222 & 1028 & \\
\hline
\end{tabular}

Table (8) demonstrated that the participants have a good level of knowledge, attitude and practices in employed, urbanized female with barely sufficient of economic and bachelor degree in age group (21-30) years, also indicated the significant relationship between (KAP) concerning COVID-19 with age, gender and level of education, while there are a significant 
relationship between knowledge and practices toward COVID-19 and monthly income at p value less than (0.05).

\section{DISCUSION}

COVID -19 has spread very quickly all over the world, including the Iraqi Kurdistan region and causing serious health issues. Kurdistan region of Iraq is a developing country and most part of the citizens is living in the urban areas. The present study was conducted to assess knowledge, attitude, and practices concerning COVID-19 in Kurdistan region of Iraq.

Regarding the socio demographic data the current study demonstrated that the age of the participants were between $(\leq 20-\leq 41)$ years old, more than half of them were female but (45.4\%) were male, lowest level of education was graduated from primary school (2.6\%) and highest rate were (46.2\%) which graduated from university and (24.4\%), (11.6\%), (11.2\%), (4\%) of them were graduated from institute, secondary school, master and doctorate degree respectively. More than half of them were from urban area, (27.7\%) were from sub urban area and only (5.1\%) of them were from rural area. The governmental employ was the highest and retired was the lowest rate of their occupation $(56.3 \%, 0.7 \%)$ respectively however $(19.4 \%$, $11.1 \%, 4.2 \%, 8.2 \%$ ) was student, self-employ, housewife, and jobless respectively. Also the highest level of their economic status were barely sufficient (65.8) and (28.9\%, 5.4\% were sufficient and insufficient respectively. The present result was disagree with the study done in Nepal and the study in Pakistan who reported that the number of male gender who participated in their study was more than female [13, 14], on the other hand, this result was in agreement with the study done in China and reported that number of female participants was more than male and nearly half of them were graduated from the university [15]. and the result about urbanization citizen in the current study was nearly similar to Pakistani study, which both of them recorded that more than half of their participants were from urban area [14]. Also the result of the current study (83.8\%) of the participants were not smoker at all and (10.9\%, 3.6\%, 1.8\%) were smoking cigarette, hooker and both of them together respectively. The result of the current study showed that the highest source of participant's knowledge was obtained from social media (36.48\%) and lowest source was obtained from Ministry of health / WHO \& CDC together. However only (2.24\%) of them were obtained from Collage of nursing and medicine.

Regarding the participant's knowledge of the present study, showed that the highest response was (77.1\%), which deals with the first action of experiencing the symptoms of COVID-19, and they preferred call health care services "122", (95.4\%) responded to immediately isolated in the quarantine when they contacted with infected person with the COVID-19, (82.2\%) of the participants said that cigarette and hookah (Shisha) smokers has more at risk of getting infected by coronavirus infection, $(50.7 \%)$ believe that the coronavirus transmitted from materials that are imported from other countries, $(66.3 \%)$ of the participants said that there are enough instructions for people who recovering from coronavirus after being discharged from the hospital, (79\%) trust that drinking alcohol not protect people from coronavirus; which is similar to the study done in Nepal and indicated that the most of their participants trusted that drinking alcohol not cure coronavirus [13]. Nearly half of the participants in the current study did not have information about transmitting coronavirus by mosquito; While worldwide organization in 2020 reported that there are no evidence or information to support that the COVID-19 could be transmitted by mosquitoes, also reported that alcohol decreases the body's immune system and increases the risk of adverse health outcomes and leads to weaken the immune system and thus decreases the ability to cope and deal with contagious diseases; so drinking too much alcohol can increase a person's risk of being infected with COVID-19 and heighten their symptoms [3]. (47.6\%) of the participants considered that the exposure to sunlight or any temperature higher than $\left(25^{\circ} \mathrm{c}\right)$ protect you from coronavirus, (K10) was dealt with the essential time of hand washing which was (after using WC, before and after meal, after touching money or animals, none of them) (95.5\%) of the participants chaos all of them. And (70.1\%) of them believed that the daily physical activity important according to ages and 
abilities during the outbreak of coronavirus; As well as (WHO, 2020) suggested that daily activity as possible is very important for every people of all ages and abilities [3].

Concerning attitude of the participants regarding COVID-19, the result of the current study revealed that (81.4\%) of them believed cleaning hands and preventing them from touching the face is the best way to reduce the risk of spreading COVID19, this result is agreed with the study done in Pakistan under the title of knowledge of COVID-19 symptoms and prevention among Pakistani adults while their participants were aware of the preventive strategies as the majority of them reported that regularly cleaning and washing hands and avoiding to touch eyes face and mouth are very important [14]. (52\%) of them trust that coronavirus is not biological war, (65\%) of them thought that keeping social distance in the community is important to the patient even after recovering from coronavirus and leaving the hospital, nearly a quarter of them believed that using masks and gloves only are not enough to keep the health from coronavirus $(72.7 \%, 74.6 \%)$ respectively, (52\%) of them were prevent publishing unpleasant and frightening news about the number of people affected and died by coronavirus from TV channels and social media, (51.8\%) of them had no information about the role of vaccines in childhood against Tuberculosis that affected by coronavirus or will be easily recovered from it, a study done in USA and they explained that COVID-19 has spread to most countries in the world; and the impact of the disease is different in different countries also they recommend that national differences in COVID-19 influence could be partially clarified by the different national policies respect to the childhood vaccination of Bacillus Calmette-Guerin (BCG). Also they detect that BCG vaccination has been reported to offer broad protection to respiratory infections. Also they found that the vaccination of BCG leads to reducing the number of reported COVID-19 cases in a country. So the combination of reduced morbidity and mortality makes BCG vaccination a potential new tool in the fight against COVID-19 [16].

Regarding the best way to protect people from coronavirus in the present study (69.8\%) of the participants believed that the advice about curfew and staying at home, (71.8) of the participants trusted that keeping people indoors by force harm them in terms of their finances, (83.8\%) of them though that washing hands must be done by a healthy way and must not less than twenty seconds, (82\%) of them believed that the health guidelines have been useful for protecting them from coronavirus.

According to practices of the participants regarding coronavirus, the present study revealed that $(70.7 \%)$ of them stayed at home and prevent visiting their relatives and friends to protect themselves and others from Coronavirus, (41.7\%) of them always used mask and gloves when they go out while $(46.2 \%, 12.1 \%)$ sometimes and never wore them respectively, as well as (79\%) of them were destroyed them after using from a suitable place, $(73.8 \%)$ of the participants washed every parts of their hands such as palm, thumb, wrist, under nails and fingers very well by soap and water when you return home, this result is different with the study done in Pakistan in 2020 which their result showed that nearly majority of their participants wore mask when they went out, however, nearly similar to the same study because the majority of them were cleaned their hands very well and performed social isolation and avoid going outside unnecessary [12].

Concerning physical activity, (61.2\%) of them sometimes were doing physical activity according to their ages and abilities when they were at home because of coronavirus, while $(17.6 \%, 21.2 \%)$ of them were doing? always and never respectively, also the result showed that only (39.2\%) were always designed a healthy suitable schedule for their daily life until they will spend their time far away from stress and fear, while (46.3\%, 14.5\%) was designed sometimes and never respectively. The current study demonstrated that only (19.5\%) of the contributors were always follow that bad and sadness news, which presented from TV and Internet regarding increasing number of death and new case of Corona virus while (54.6\%, 25.9\%) were followed sometimes and never respectively. (62\%) of them always prevented hand shaking with everybody to protect themselves and others from corona virus but (32.9\%, $5.1 \%)$ of them were prevented sometimes and never respectively. Also the study indicated that (62.3\%) were always washed hand by soap and water or alcohol immediately when they 
cough or sneeze on their hand and (31.5\%) of them washed their hands sometimes while still (6.2\%) never washed their hands. And the study also demonstrated that only $(13.2 \%)$ of the participants were always injected the flu vaccination against seasonal flu annually while $(58.3 \%, 28.5 \%)$ of them were never and sometimes vaccinated. Also the results of the present study demonstrated a strong positive correlation among knowledge, attitude, and practice.

The results of the current study were showed that there were no significant relationship between knowledge regarding COVID-19 and age, gender, residential area at p value greater than 0.05 except educational level, occupation, and monthly income. Also there was no significant relationship between attitude concerning coronavirus and age, educational level and occupation at $\mathrm{p}$ value greater than 0.05 except gender, residential area and monthly income at $\mathrm{p}$ value less than 0.05 . As well as the present study revealed the significant relationship between practices regarding COVID-19 and all socio-demographic such as age, gender, residential area, level of education, occupation and monthly income at $\mathrm{p}$ value less than 0.05 .

Also the result of the current study demonstrated that the participants had a good level of knowledge, attitude and practices regarding COVID-19; this finding was supported by the study done in China and another study in India concerning KAP regarding COVID-19 and they reported that the majority of their participants had a good level of knowledge, attitude and practice regarding COVID-19 $[15,17]$. On other hand, this result was disagreed with the study done in Nepal which indicated that the knowledge among their people toward COVID19 is satisfactory and also the study mentioned a significant number of their participants are lacking confidence when compared to other countries [13].

\section{CONCLUSION}

The participants obtained knowledge regarding COVID-19 from social media more from World Health Organization, Center of Disease Control, and Prevention and ministry of health, the results of the current study were indicated that the knowledge, attitude, and practices are in suitable level when compared to other developed countries. Kurdistan region is facing a stiff challenge to control the spread of COVID-19 among its population. Also the participants were safely practiced such as not going to crowded places, hand washing as WHO and CDC guidelines suggested. The study recommended to present or spread healthy scientific issues to improve the knowledge of the Kurdish citizens because they are much more dependable scientifically than social media toward COVID-19 to protect themselves and rapid transmission of the disease and could be controllable for the serious situation.

\section{REFERENCE}

[1] C. P. E. R. E. Novel, "The epidemiological characteristics of an outbreak of 2019 novel coronavirus diseases (COVID-19) in China," Zhonghua liu xing bing xue za zhi= Zhonghua liuxingbingxue zazhi, vol. 41, no. 2, p. 145, 2020, doi: 10.3760/cma.j.issn.0254-6450.2020.02.003.

[2] N. Chen et al., "Epidemiological and clinical characteristics of 99 cases of 2019 novel coronavirus pneumonia in Wuhan, China: a descriptive study," The Lancet, vol. 395, no. 10223, pp. 507-513, 2020, doi: 10.1016/S0140-6736(20)30211-7.

[3] W. H. Organization, "Coronavirus disease (COVID-2019) situation reports," ed: World Health Organization, 2020.

[4] M. O. Nour, A. O. Babilghith, H. A. Natto, F. O. Al-Amin, and S. M. Alawneh, "Knowledge, attitude and practices of healthcare providers towards MERS-CoV infection at Makkah hospitals, KSA," International Research Journal of Medicine and Medical Sciences, vol. 3, no. 4, pp. 103-12, 2015.

[5] M. Cetron and P. Simone, "Battling 21st-century scourges with a 14th-century toolbox," Emerging infectious diseases, vol. 10, no. 11, p. 2053, 2004, doi: 10.3201/eid1011.040797_12.

[6] K.-T. Goh et al., "Epidemiology and control of SARS in Singapore," Annals-Academy of Medicine Singapore, vol. 35, no. 5, p. 301, 2006.

[7] M. Cetron and J. Landwirth, "Public health and ethical considerations in planning for quarantine," The Yale journal of biology medicine, vol. 78, no. 5, p. 329, 2005.

[8] C. f. D. C. a. P. (CDC), "Interim Guidance on Management of Coronavirus Disease 2019 (COVID-19) in Correctional and Detention Facilities," Centers for Disease Control and Prevention (CDC), accessed July 19, 2020 2020. [Online]. Available: https://www.cdc.gov/coronavirus/2019-ncov/community/correctiondetention/guidance-correctional-detention.html. 
[9] A. Wilder-Smith and D. O. Freedman, "Isolation, quarantine, social distancing and community containment: pivotal role for old-style public health measures in the novel coronavirus (2019-nCoV) outbreak," Travel medicine, vol. 27, no. 2, p. taaa020, 2020, doi: 10.1093/jtm/taaa020.

[10] T. Day, A. Park, N. Madras, A. Gumel, and J. Wu, "When Is Quarantine a Useful Control Strategy for Emerging Infectious Diseases?," American Journal of Epidemiology, vol. 163, no. 5, pp. 479-485, 2006, doi: 10.1093/aje/kwj056 \%J American Journal of Epidemiology.

[11] U. N. O. f. t. C. o. H. Affairs, "IRAQ: COVID-19 Situation Report No.6," Situation Report, 17 Mar 2020 2020. [Online]. Available: Accessed at https://reliefweb.int/report/iraq/iraq-covid-19-situation-report-no-617-march-2020.

[12] K. R. Government, "Latest information about Coronavirus (COVID-19)," GOV.KRD, accessed July 26, 2020 2020. [Online]. Available: https://gov.krd/coronavirus/dashboard/.

[13] A. Hussain, T. Garima, B. M. Singh, R. Ram, and R. P. Tripti, "Knowledge, attitudes, and practices towards COVID-19 among Nepalese Residents: A quick online cross-sectional survey," Asian Journal of Medical Sciences, vol. 11, no. 3, pp. 6-11, 2020, doi: 10.3126/ajms.v11i3.28485.

[14] S. Malik, "Knowledge of COVID-19 Symptoms and Prevention Among Pakistani Adults: A Crosssectional Descriptive Study," PsyArXiv, 2020, doi: 10.31234/osf.io/wakmz.

[15] B.-L. Zhong et al., "Knowledge, attitudes, and practices towards COVID-19 among Chinese residents during the rapid rise period of the COVID-19 outbreak: a quick online cross-sectional survey," International Journal of Biological Sciences, vol. 16, no. 10, p. 1745, 2020, doi: 10.7150/ijbs.45221.

[16] A. Miller, M. J. Reandelar, K. Fasciglione, V. Roumenova, Y. Li, and G. H. Otazu, "Correlation between universal BCG vaccination policy and reduced morbidity and mortality for COVID-19: an epidemiological study," MedRxiv, 2020, doi: 10.1101/2020.03.24.20042937.

[17] B. S. Tomar et al., "Indian communitys Knowledge, Attitude \& Practice towards COVID-19," MedRxiv, 2020, doi: 10.1101/2020.05.05.20092122. 